\section{SEND YOUR PATIENTS TO A MORE PEACEFUL PLACE}

Using the ZEISS cinemizer OLED head-mounted display, a visit to the dentist can be a much calmer and less nerve-wracking experience for patients. The video glasses are lightweight and ergonomically designed to fit more people, from young children to adults.

As many as 75\% of adult patients experience some degree of anxiety when visiting the dentist. One of the most effective and natural ways of putting your patients at ease is through distraction, which can take the mind's attention away from the sights and sounds of the dental office to a calmer and more peaceful place.

Once the dentist slips the cinemizer OLED on the patient, films, cartoons or calming scenes with gentle music can be played. The patient is immersed in the experience, and distracted from potentially stressful treatments, making it easier for the dentist to do their work quickly and efficiently.

The cinemizer can take a video source from HDMI, or an iPod, so is very convenient to use. In some countries there are also packages specific to dentistry available. These solutions offer an all-in-one package specifically designed for use in dental surgeries.

\section{GUARDING AGAINST NIGHT-TIME GRINDING}

DenTek's Custom Comfort Dental Guard protects against night time bruxism by cushioning the teeth and keeping them apart, with the least material possible and in the most natural position.

By preventing the upper and lower jaw from touching, a dental guard helps prevent damage to teeth, prevent noise associated with grinding, and associated symptoms like jaw pain, headaches and sleepless nights.

DenTek goes beyond brushing, providing innovative oral care products that clean between the teeth, promoting oral hygiene habits for a fabulous smile and good overall physical health. DenTek's Beyond Brushing range also includes floss picks, interdental brush cleaners, disposable picks and tongue cleaners.

The DenTek Custom Comfort Dental Guard is

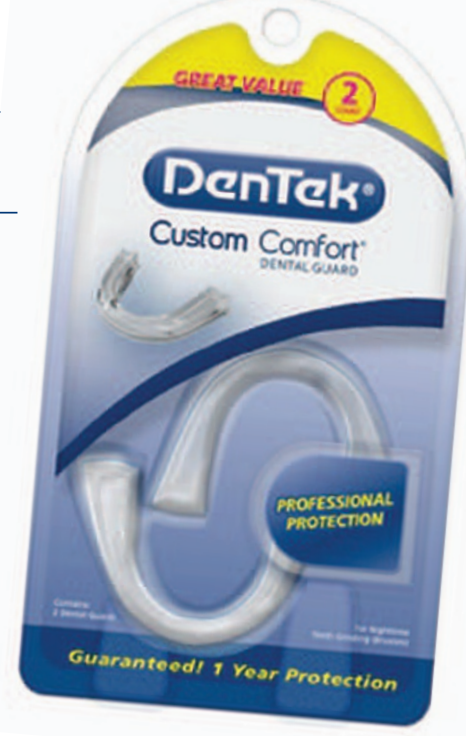

available from various retailers for RRP £25.90. For more information visit www.dentekuk. co.uk.

\title{
SAFE, COST-EFFECTIVE MARKETING FOR YOUR PRACTICE
}

Toothpick is the UK's first nationwide online dental appointment booking service, letting users find, compare and book local dentists. Toothpick. com will roll out across the UK this year and so far over 1,000 dentists have booked in 20,000 dental appointments online.

Toothpick has created an online directory of UK dentists, allowing people to find local providers, compare price and service and book online. It offers people cost savings, convenience and better service in their dental bookings and can even be used to find emergency appointments. Users can also choose whether they want to search for NHS or private practices, giving them the broadest possible choice of provider.

Toothpick has been developed in partnership with Henry Schein, the world's largest provider of healthcare products to dental practitioners, meaning that it is safe, secure and reliable. The product is closely integrated with the company's dental appointment scheduling software, which is in use by more than 10,000 dentists across the UK.

Toothpick operates a subscription model for dentists, charging them a low monthly fee to be featured on the service. It gives dentists access to new patients and can help to fill empty, late notice appointment slots.

\section{CLEAN, LUBRICATE AND STERILISE WITH EASE}

Dental practices can meet best practice requirements for instrument decontamination in as little as 60 minutes with this three-step solution from Prestige Medical. The reduction in processing time results in significant cost and time savings at a time

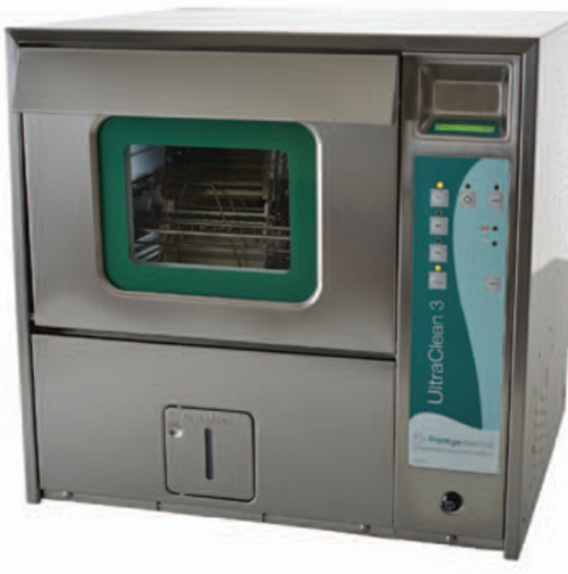
when practice incomes are being squeezed. Step One Clean: The new UltraClean 3 Washer Disinfector features a 35 minute 'Quick
Cycle' with validated cleaning results. Available in either bench top or under bench models, UltraClean 3 also features a glass viewing pane and illuminated chamber.

Step Two - Lubricate: The new Optimax Hand Piece Lubrication System is fast and simple to use, lubricating up to three hand pieces in less than two minutes. Air activated, the Optimax automatically rotates the hand pieces, ensuring correct lubrication every time.

Step Three - Sterilise: Optima $\mathrm{B}$ is the fastest B class vacuum autoclave on the market and can sterilise a full $6 \mathrm{~kg}$ load in only 23 minutes including drying. Now it also features a new handpiece cycle which can sterilise up to 12 handpieces in only 15 minutes.

All products are "plug and play' - supplied complete with all the accessories you need to get you started. UltraClean 3 and Optima will be installed and commissioned by Prestige Medical's own engineers who will also teach you how to use the equipment.

Visit www.prestigemedical. co.uk/uk/dentistry. 